\title{
Echo Metadata Model: desarrollo de un modelo de metadatos para la descripción de los materiales audiovisuales
}

\author{
Ana Belén Ríos Hilario \\ Universidad de Salamanca (España)
}

\section{Resumen}

Se realiza un análisis comparativo entre el esquema de metadatos ECHO, diseñado para la descripción del material audiovisual de las filmotecas históricodocumentales, y el modelo entidad-relación (E-R) propuesto por la IFLA en su obra Functional Requeriments Bibliographic Records (FRBR). En primer lugar, y tras una breve introducción en la que se destaca la importancia que tiene la aplicación del patrón E-R para el desarrollo conceptual de sistemas de bases de datos relacionales, se definen los FRBR centrándonos en la explicación de la metodología que utiliza y en la descripción de las entidades que define, resaltando especialmente las entidades del grupo 1 -obra, expresión, manifestación e ítem—ya que estas son el núcleo de tal trabajo y de su metodología. A continuación se define el ECHO Metadata Model como un modelo abierto e interoperable capaz de representar los múltiples aspectos de los recursos audiovisuales y, tras describir su proceso de creación y desarrollo, se establecen las semejanzas y diferencias con la obra que se ha tomado como referente. Como conclusión, se puede decir que básicamente el esquema ECHO se adapta al modelo propuesto por la IFLA, ya que la definición de nuevas entidades y relaciones no es en sí misma una diferencia, sino que entra dentro de lo que debe ser la aplicación e implementación del informe de la IFLA en la descripción de los distintos tipos de recursos, en este caso concreto del material audiovisual.

Palabras clave: Descripción materiales audiovisuales. Echo Metadata Model. FRBR.

\section{Abstract}

A comparative analysis between the ECHO metadata scheme and the Functional Requirements Bibliographic Records (FRBR) is made. First, the importance that the application of the entity-relation model (E-R) for the conceptual development of relational databases is stated. Second, the FRBR - proposed by the International Federation of Library Associations (IFLA) - are defined, with a 
special attention to the group 1 entities - work, expression, manifestation and item. Third, the ECHO Metadata Model, designed for the description of audiovisual materials in film archives, is reviewed and analyzed. It is concluded that the ECHO Metadata Model is compatible with the FRBR for the description of audiovisual materials.

Keywords: Audiovisual material description. ECHO Metadata Model. FRBR.

\section{Introducción}

El modelo entidad-relación (E-R), basado en la definición de entidades, atributos y relaciones, es uno de los más empleados en la actualidad en numerosas aplicaciones que tienen como objetivo el desarrollo conceptual de sistemas de bases de datos relacionales. En nuestro campo, es significativa la aplicación de tal esquema para la elaboración por parte de la IFLA de la obra Functional Requirements for Bibliographic Records (FRBR). Este documento, válido para distintos tipos de materiales (como, por ejemplo, el cartográfico o el musical), se ha convertido en un referente, de tal manera que pronto han surgido distintos modelos que, partiendo de él, lo han adaptado a casos específicos, como puede ser el INDECS, cuyo objetivo son los sistemas comerciales por Internet, o incluso modelos que van más allá y que tienden a armonizar los diferentes proyectos; tal es el caso del ABC. Entre todos ellos, nosotros hemos optado por estudiar el modelo de metadatos ECHO aplicado a los materiales audiovisuales. Tal elección no ha sido fortuita y responde a varios motivos. En primer lugar, nos parecía atrayente al centrarse en este tipo de recursos clave en la época en la que vivimos, pero a su vez poco estudiados si lo comparamos con el material textual. Por otro lado, es también un modelo de metadatos, tal y como puede extraerse de su propia denominación, y una aplicación del informe de la IFLA anteriormente citado. A estas alturas nadie pone en duda la validez y repercusiones que los metadatos han tenido para describir los objetos de información de un entorno en red y, aunque este no va a ser el tema central de nuestro estudio, reafirman la importancia del modelo ECHO.

Partiendo de tales premisas, nuestro objetivo es la realización de un análisis comparativo entre el modelo de la IFLA y el ECHO, para reflejar cómo se ha adaptado el primer esquema a un modelo de metadatos realizado específicamente para los materiales audiovisuales.

\section{El modelo FRBR}

En 1998 tiene lugar la publicación por parte de la IFLA del estudio denominado Functional Requirements Bibliographic Record (FRBR). Este modelo ha recibido un consenso unánime, concibiendo el registro bibliográfico como una agregación de datos que son asociados con las entidades descritas en los catálogos bibliotecarios y en las bibliografías nacionales. Los datos pertenecen a los mate- 
riales textuales, musicales, cartográficos, gráficos y tridimensionales y, por supuesto, a los audiovisuales.

La metodología usada en este estudio, basada en los objetivos del mismo, aplica la técnica del análisis de entidad, que se usa en el desarrollo de los modelos conceptuales para los sistemas de bases de datos relacionales. Esta técnica comienza por aislar las entidades que son objeto de interés clave para los usuarios de los registros bibliográficos. Posteriormente, se identifican las características o atributos asociados con cada entidad y las relaciones entre entidades, que son las más importantes para los usuarios al formular las búsquedas bibliográficas, interpretar las respuestas a estas búsquedas y "navegar" por el universo de entidades descritas en los registros bibliográficos.

Las entidades definidas en el informe de la IFLA representan los objetos clave fruto de los intereses de los usuarios de los registros bibliográficos. Estas entidades se dividen en tres grandes grupos. El grupo 1 comprende los productos de los esfuerzos intelectuales o artísticos que se nombran o describen en los registros bibliográficos: obras, expresiones, manifestaciones e ítems (1). Forman la base del modelo y de su metodología. El grupo 2 engloba aquellas entidades responsables del contenido intelectual o artístico, de la producción física y de la difusión, y de la conservación de tales productos: personas y entidades corporativas. También pueden funcionar como materias. El grupo 3 incluye un conjunto adicional de entidades que sirven como materias de los esfuerzos intelectuales o artísticos: concepto, objeto, suceso y lugar. Estas entidades frecuentemente se emplean como materias de las obras. También pueden referirse a una única obra o a multitud de ellas, y cada obra puede incluir varias entidades del tercer grupo.

A continuación desarrollaremos de modo más detenido las entidades del grupo 1, ya que son las principales y sirven de apoyo al modelo ECHO. Obra se define como "una creación inequívoca intelectual o artística" (IFLA Study Group on the Funcional Requirements for Bibliographic Records, 1998, p. 16). Es una entidad abstracta, no un objeto material. Se reconoce a través de las realizaciones o expresiones individuales de la misma, pero la obra propiamente dicha solamente existe en la similitud del contenido entre las varias expresiones de la misma. La expresión es "la realización intelectual o artística de una obra" (ibidem, p. 18). Comprende las palabras, frases, párrafos, etcétera, específicos, que resultan de la realización o expresión de una obra, y provee una distinción en el contenido intelectual entre una realización y otra de la misma obra. Sin embargo, las diferencias en la tipografía o en el papel no constituyen una nueva expresión. La manifestación es "la representación física de la expresión de una obra" (ibidem, p. 20). En otras palabras, es la publicación de la expresión. Finalmente, el ítem es "un único ejemplar de una manifestación" (ibidem, 1998, p. 23).

Scire. $11: 2$ (sep.-dic. 2005) 133-139. ISSN 1135-3761. 
El siguiente ejemplo aclara e ilustra las entidades anteriormente definidas:

- $\mathrm{O}_{1}$ Playback, de Ronald Hayman

- $\mathrm{E}_{1}$ el texto del autor editado para una publicación

- $\mathrm{M}_{1}$ el libro publicado en 1973 por Davis-Poyter

- $\mathrm{I}_{1}$ la copia autografiada por el autor

\section{ECHO Metadata Model}

El objetivo del ECHO Metadata Model es definir un nuevo modelo abierto e interoperable capaz de representar los múltiples aspectos de los recursos audiovisuales. La definición del modelo se llevó a cabo siguiendo una metodología particular que constaba de varias etapas. En primer lugar, se realizó un pequeño estudio para obtener una visión general de los modelos de metadatos utilizados actualmente, como por ejemplo el MPEG 7 (Moving Picture Expert Group). Sin embargo, no es nuestra intención estudiar el modelo desde el punto de vista de los metadatos, sino de la aplicación por parte del mismo del modelo de la IFLA propuesto anteriormente. Por lo tanto, a la hora de describirlo lo haremos desde esta última perspectiva.

Posteriormente, mediante una serie de encuestas se intentó conocer "los deseos de datos" por parte de los miembros del ECHO. En concreto podemos decir que el modelo de metadatos propuesto por el ECHO Project es el resultado del análisis de tres cuestionarios diferentes hechos a medida para archiveros de filmotecas histórico-documentales, proveedores tecnológicos e investigadores. Su estructura está de acuerdo con el modelo de la IFLA. Este modelo, como ya hemos visto, define cuatro entidades —obra, entidades, manifestación e ítem-, que describen los diferentes aspectos del recurso. Estas cuatro entidades están implícitamente distribuidas en cuatro niveles de descripción que aparecen explícitos en el modelo ECHO. Los campos de metadatos relativos a la descripción semántica se localizan en lo alto del nivel más abstracto, denominado nivel obra. Como la misma obra intelectual o artística pueden representarse de diferentes modos (por ejemplo, el mismo documento puede tener una traducción al español, una versión subtitulada, etcétera), se incluye un nivel particular, denominado nivel expresión, que describe las diferentes versiones de la misma obra. Además, la misma versión de una obra puede almacenarse en formatos y soportes diferentes. Estos se describen en el nivel manifestación. Finalmente, las informaciones de los materiales individuales de la misma manifestación se mantienen en el nivel ítem.

El modelo ECHO (fig. 1) se obtuvo añadiendo a cada nivel algunas entidades especializadas para los documentos de los que trata el propio proyecto. En el nivel obra se introdujo la entidad documento audiovisual (avdocument). Se trata de una entidad genérica que representa a una obra intelectual o artística audiovisual. Cada 
documento, que pertenece a las entidades del nivel obra, puede tener una o más versiones. Consecuentemente, se añadió la entidad versión en el nivel expresión. Por ejemplo, un elemento de versión puede ser la realización audio de una cinta de noticias; otro, la transcripción de la misma cinta. Esto se expresa en el modelo por medio de la expresión ExpresadoPor (ExpressedBy) entre documento audiovisual y versión. Se ha realizado una descomposión de versión en vídeo, audio y trans-

Deliverable 3.1.1

WORK LEVEL

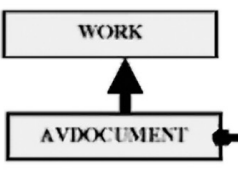

ExpressediBy

ตั

EXPRESSION LEVEL

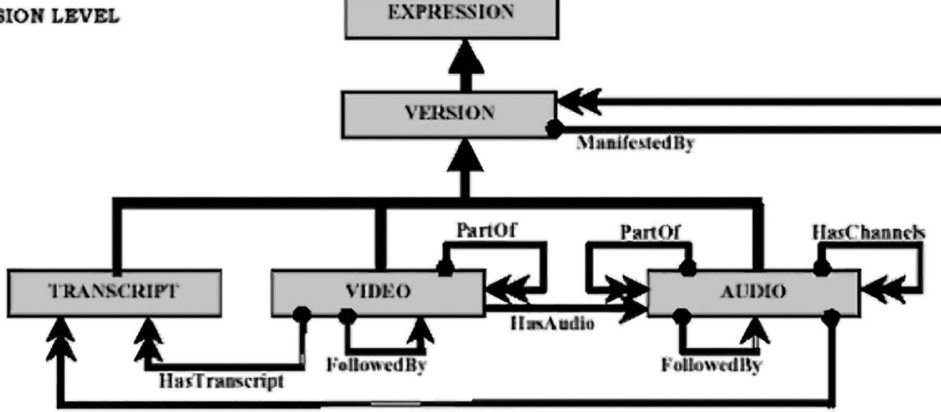

Has'Transcript
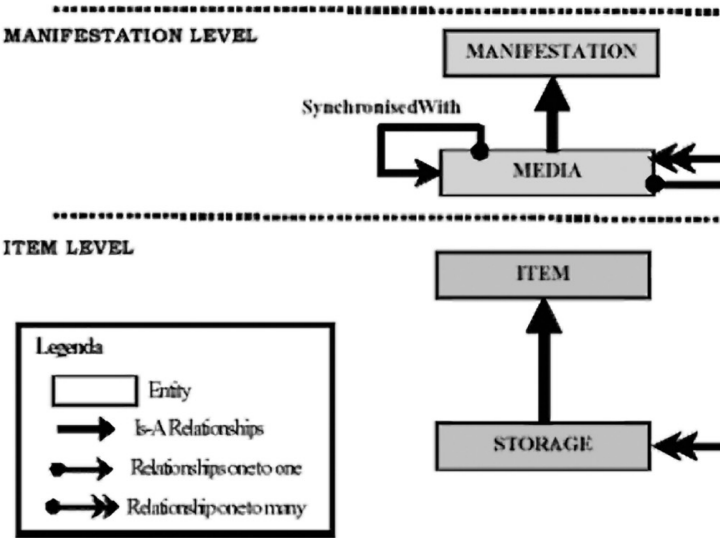

AvgilableAs

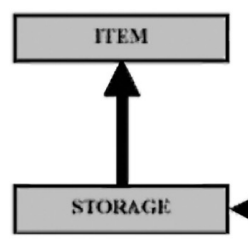

Figura 1. Representación del modelo ECHO

Scire. $11: 2$ (sep.-dic. 2005) 133-139. ISSN 1135-3761. 
cripción, ya que cada uno de estos tipos particulares de versión se caracteriza por su propio conjunto de campos de metadatos. La relación ParteDe (PartOf), tanto de la entidad vídeo como de audio, permite asociar una parte de un documento con el documento global, mientras que la relación SeguidoPor (FollowedBy) puede usarse para expresar la sucesión temporal de las partes del documento y el tipo de transición entre una parte y la siguiente. Si se describe un documento sonoro, la relación TieneCanales (HasChannels) se define para hacer constar los diferentes canales de sonido que tiene un documento sonoro. De tal modo que, si se describe un documento audiovisual, la parte sonora se describe en la entidad audio y la parte visual se describe en la entidad vídeo. La relación TieneSonido (HasAudio) crea el enlace entre dos partes del mismo documento audiovisual, mientras que la relación TieneTranscripción (HasTranscript) enlaza el documento vídeo con la transcripción de su sonido (en el caso de que se haya perdido el documento sonoro de ese vídeo) o el documento sonoro con su transcripción. En el nivel manifestación, la entidad medios (media) representa las clases diferentes de soportes digitales que pueden usarse para mantener las versiones de los documentos. Cada versión del nivel expresión puede almacenarse en uno o más media describiéndose por medio de la relación ManifestadoPor (ManifestedBy). La sincronización entre los diferentes elementos de medios se realiza gracias a la relación SincronizadoCon (SynchronisedWith). Finalmente, la entidad almacenamiento (storage) en el nivel ítem describe la información general acerca de las copias individuales de los documentos. La relación DisponibleComo (AvailableAs) crea enlaces entre cada elemento de la entidad medios y uno o más elementos en la entidad almacenamiento.

Para cada una de estas entidades se han definido campos de metadatos que se describen en el anexo A del propio modelo.

\section{Conclusiones}

A través de la propia definición del modelo ECHO hemos ido viendo su adaptación al propuesto por la IFLA y, como hemos podido comprobar, las diferencias vienen marcadas por las características del propio material audiovisual. Estas distinciones ya han sido expuestas, por lo que no las vamos a volver a recalcarlas.

Sin embargo, sí que nos gustaría destacar el hecho de la metodología empleada en el modelo ECHO, especialmente el estudio de usuarios previo a la realización del esquema empleando el método de los cuestionarios. La ausencia de un estudio de este tipo es una de las principales objeciones que recibe el estudio FRBR.

\section{Notas}

(1) El término ítem generalmente se traduce al español como 'publicación'. Sin embargo, en este caso la palabra tiene un significado más específico, el de 'publicación única', 'ejemplar único'. 


\section{Referencias}

Amato, Giuseppe; Castelli, Donatella; Pisani, Serena (2000). A metadata model for historical documentary films. // Proceedings of the 4th European Conference ECDL 2000 (Lisboa, sept. 2000).

Amato, Giuseppe; Castelli, Donatella; Pisani, Serena; Venerosi, Paola; Poncin, Philippe; Vinet, Laurent (2000). Metadata modelling report. [Pisa]: [Consiglio Nazionale delle Ricerche, Istituto di Elaborazione della Informazione (IEI-CNR)], 2000. URL: $<$ http://pc-erato2.iei.pi.cnr.it/echo/public/deliv/D3-1-1\%20ECHO\%20Metadata \%20Modelling.pdf>. Consultado: 2004-01-12.

Amato, Giuseppe; Gennaro, Claudio; Savino, Pasquale (2001). Searching documentary films on line: the ECHO Digital Library // Proceedings of the 6th ICHIM Conference (Milán, 3-7 sept. 2001). S. 1.: s. n., 2001. URL: <http://faure.iei.pi.cnr.it/ gennaro/download/ ags_ichimo1.pdf >. Consultado: 2004-01-12.

IFLA Study Group on the Funcional Requirements for Bibliographic Records (1998). Functional Requirements for Bibliographic Records: final report. Múnich: Saur. URL: $<$ http//www.ifla.org./VII/s13/frbr/frbr.htm>. Consultado: 2002-09-20.

Méndez Rodríguez, Eva (2002). Metadatos y recuperación de la información: estándares, problemas y aplicabilidad en bibliotecas digitales. Gijón: Trea, 2002.

Ríos Hilario, Ana Belén (2003). La estructura conceptual del registro bibliográfico. // Ríos Hilario, Ana Belén. Nuevos horizontes en el análisis de los registros y la normativa bibliográfica. Gijón: Trea, 2003.

Scire. 11 : 2 (sep.-dic. 2005) 133-139. ISSN 1135-3761. 